\title{
ON c-NORMALITY OF FINITE GROUPS
}

\author{
M. ASAAD and M. EZZAT MOHAMED
}

(Received 1 September 2001; revised 22 September 2003)

Communicated by R. Howlett

\begin{abstract}
A subgroup $H$ of a finite group $G$ is said to be c-normal in $G$ if there exists a normal subgroup $N$ of $G$ such that $G=H N$ with $H \cap N \leq H_{G}=$ Core $_{G}(H)$. We are interested in studying the influence of the $c$-normality of certain subgroups of prime power order on the structure of finite groups.
\end{abstract}

2000 Mathematics subject classification: primary 20D10, $20 \mathrm{D} 30$.

\section{Introduction}

All groups in this paper will be finite. We say, following Wang [11], that a subgroup $H$ of $G$ is $c$-normal in $G$ if there exists a normal subgroup $N$ of $G$ such that $G=H N$ with $H \cap N \leq H_{G}$, where $H_{G}=\operatorname{Core}_{G}(H)=\bigcap_{g \in G} H^{g}$ is the maximal normal subgroup of $G$ which is contained in $H$.

Two subgroups $H$ and $K$ of $G$ are said to permute if $H K=K H$. We say, following Kegel [9], that a subgroup of $G$ is $S$-quasinormal in $G$ if it permutes with every Sylow subgroup of $G$.

Let $p$ be a prime and let $P$ be a $p$-subgroup of $G$, we write

$$
\Omega(P)= \begin{cases}\Omega_{1}(P) & \text { if } p>2 \\ \Omega_{2}(P) & \text { if } p=2,\end{cases}
$$

where $\Omega_{i}(P)$ is the subgroup of $P$ generated by its elements of order dividing $p^{i}$.

Let $\mathfrak{I}$ be a class of groups. We call $\mathfrak{\Im}$ a formation if $\mathfrak{I}$ contains all homomorphic images of a group in $\Im$, and if $G / M$ and $G / N$ are in $\Im$, then $G /(M \cap N)$ is in $\Im$

() 2005 Australian Mathematical Society $1446-7887 / 05 \$ A 2.00+0.00$ 
for normal subgroups $M, N$ of $G$. Each group $G$ has a smallest normal subgroup $N$ such that $G / N$ is in $\Im$. This uniquely determined normal subgroup of $G$ is called the $\Im$-residual subgroup of $G$ and will be denoted by $G^{\Im}$. A formation $\Im$ is said to be saturated if $G / \Phi(G) \in \mathfrak{I}$ implies $G \in \mathfrak{I}$. Throughout this paper $\mathfrak{U}$ will denote the class of supersolvable groups. Clearly, $\mathfrak{U}$ is a formation. Since a group $G$ is supersolvable if and only if $G / \Phi(G)$ is supersolvable [6, VI, page 713], it follows that $\mathfrak{U}$ is saturated.

With every prime $p$ we associate some formation $\Im(p)(\Im(p)$ could possibly be empty). We say that $\Im$ is the local formation, locally defined by $\{\Im(p)\}$ provided $G \in \mathfrak{I}$ if and only if for every prime $p$ dividing $|G|$ and every $p$-chief factor $H / K$ of $G$, Aut $_{G}(H / K) \in \Im(p)\left(\operatorname{Aut}_{G}(H / K)\right.$ denotes the group of automorphisms induced by $G$ on $H / K$ and it is isomorphic to $G / C_{G}(H / K)$ ). It is known (see [5, IV, 4.6]) that a formation is saturated if and only if it is local.

We assume throughout that $\Im$ is a formation, locally defined by the system $\{\Im(p)\}$ of full and integrated formations $\Im(p)$ (that is, $S_{p} \Im(p)=\Im(p) \subseteq \Im$ for all primes $p$, where $S_{p}$ is the formation of all finite $p$-groups). It is well known (see [5, IV, 3.7]) that for any saturated formation $\Im$, there is a unique integrated and full system which locally defines $\Im$.

A solvable normal subgroup $N$ of a group $G$ is an $\Im$-hypercentral subgroup of $G$ (see Huppert [7]) provided $N$ possesses a chain of subgroups $1=N_{0} \triangleleft N_{1} \triangleleft \cdots \triangleleft N_{r}=N$ satisfying (i) every factor $N_{i+1} / N_{i}$ is a chief factor of $G$, and (ii) if $N_{i+1} / N_{i}$ has order a power of the prime $p_{i}$, then $G / C_{G}\left(N_{i+1} / N_{i}\right) \in \Im\left(p_{i}\right)$. The product of all $\Im$-hypercentral subgroups of $G$ is again an $\Im$-hypercentral subgroup of $G$, denoted by $Z_{\mathfrak{Y}}(G)$ and called the $\Im$-hypercentre of a group $G$.

Ito in [8], proved that a group $G$ of odd order is nilpotent provided that every subgroup of $G$ of prime order lies in the center of $G$. Wang [11], proved that if all subgroups of $G$ of prime order or order 4 are c-normal in $G$, then $G$ is supersolvable. Deyu and Xiuyun [4], proved the following: (i) If $K$ is a normal subgroup of a solvable group $G$ of odd order such that $G / K$ is supersolvable and all subgroups of Fit $(K)$ of prime order are c-normal in $G$, then $G$ is supersolvable. (ii) If $K$ is a normal subgroup of a solvable group $G$ such that $G / K$ is supersolvable and all maximal subgroups of all Sylow subgroups of $\operatorname{Fit}(K)$ are c-normal in $G$, then $G$ is supersolvable.

The aim of this paper is to improve and extend the above mentioned results in [4]. The results of our paper are obtained by independent proofs to those in [4].

Our notation is standard and taken mainly from [5].

\section{Preliminary results}

LEMMA 2.1. Let $H \leq K \leq G$. 
(i) If $H$ is c-normal in $G$, then $H$ is c-normal in $K$.

(ii) If $H$ is a normal subgroup of $G$, then $K$ is c-normal in $G$ if and only if $K / H$ is c-normal in $G / H$.

ProOF. See [11, Lemma 2.1, page 956].

LEMMA 2.2. Let $P$ be a normal $p$-subgroup of $G$ and let $Q$ be a $q$-subgroup of $G$ such that $p \neq q$. If $Q$ is c-normal in $G$ then $Q P / P$ is c-normal in $G / P$.

ProOF. See [13, Lemma 2.4].

LEMMA 2.3. Let $p$ be the smallest prime dividing $|G|$ and let $P$ be a Sylow $p$ subgroup of $G$. If all subgroups of $P$ of order $p$ or order 4 are $S$-quasinormal and, in particular normal, in $G$, then $G$ is $p$-nilpotent.

Proof. See [10, Theorem 3.2, page 290].

LEMMA 2.4. Let $K$ be a normal subgroup of $G$ such that $G / K \in \Im$, where $\Im$ is a saturated formation. If $\Omega(P) \leq Z_{\Im}(G)$, where $P$ is a Sylow $p$-subgroup of $K$, then $G / O_{p^{\prime}}(K) \in \Im$.

Proof. See [3, Theorem, page 2].

LEMMA 2.5. If $G$ is a solvable group and all subgroups of Fit $(G)$ of prime order or order 4 are $S$-quasinormal and, in particular normal, in $G$, then $G$ is supersolvable.

Proof. See [2, Corollary 2, page 402].

LEMMA 2.6. If $\Im$ is a saturated formation and $N$ is an $\Im$-hypercentral subgroup of $G$, then $G / C_{G}(N) \in \Im$.

Proof. This is an easy consequence of a result due to Huppert (see [5, IV, 6.10]).

LEMMA 2.7. Let $\mathfrak{I}$ be a saturated formation containing $\mathfrak{U}$. Suppose that $G$ is a solvable group with a normal subgroup $K$ such that $G / K \in \Im$. If all maximal subgroups of all Sylow subgroups of Fit $(K)$ are $S$-quasinormal and, in particular normal, in $G$, then $G \in \Im$.

Proof. See [1, Theorem 1.4, page 3650].

LEMMA 2.8. Let $P$ be a normal $p$-subgroup of $G$. If $P \cap \Phi(G)=1$, then $P$ is $a$ direct product of abelian minimal normal subgroups of $G$.

PROOF. See [5, Theorem 10.6, page 36]. 


\section{Main results}

We begin with the following lemma:

Lemma 3.1. Let $p$ be the smallest prime dividing $|G|$ and let $P$ be a Sylow $p$ subgroup of $G$. If all subgroups of $P$ of order $p$ or order 4 are c-normal in $G$, then $G$ is p-nilpotent.

PROOF. We prove the result by induction on $|G|$. If all subgroups of $P$ of order $p$ or order 4 are normal in $G$, then $G$ is $p$-nilpotent by Lemma 2.3. Thus, we may assume that there exists a subgroup $H$ of $P$ of order $p$ or order 4 such that $H$ is not normal in $G$. By hypothesis, $H$ is c-normal in $G$. Then there exists a normal subgroup $N$ of $G$ such that $G=H N$ with $H \cap N \leq H_{G}$, and since $H$ is not normal in $G$, it follows that $N<G$. Clearly, $P \cap N$ is a Sylow $p$-subgroup of $N$. By Lemma 2.1 (i), all subgroups of $P \cap N$ of order $p$ or order 4 are c-normal in $N$. Then, by induction on $|G|, N$ is $p$-nilpotent and so also does $G$.

REMARK. The formation $\mathfrak{U}$ of all supersolvable groups is locally defined by the integrated and full system $\{\mathfrak{U}(p)\}$, where for each prime $p, \mathfrak{U}(p)$ is the class of all strictly $p$-closed groups (see [12, Theorem 1.9 and Corollary 1.5]). (Let $p$ be a prime. A group $G$ is said to be strictly $p$-closed whenever $P$, a Sylow $p$-subgroup of $G$, is normal in $G$ with $G / P$ abelian of exponent dividing $p-1$.)

We can now prove:

THEOREM 3.2. Let $\Im$ be a saturated formation containing $\mathfrak{U}$ and let $G$ be a group. Then the following two statements are equivalent:

(i) $G \in \mathfrak{I}$.

(ii) There exists a normal subgroup $K$ in $G$ such that $G / K \in \Im$ and all subgroups of $K$ of prime order or order 4 are c-normal in $G$.

ProOF. (i) implies (ii): If $G \in \Im$, then (ii) is true with $K=1$.

(ii) implies (i): Suppose the result is false and let $G$ be a counterexample of minimal order. By Lemma 2.1 (i) and Lemma 3.1, $K$ possesses an ordered Sylow tower and so $K$ has a normal Sylow $p$-subgroup $P$, where $p$ is the largest prime dividing $|K|$. Clearly, $P$ is a normal $p$-subgroup of $G$ and so $(G / P) /(K / P) \cong G / K \in \Im$. By Lemma 2.2, all subgroups of $K / P$ of prime order or order 4 are c-normal in $G / P$. Then, by the minimality of $G, G / P \in \Im$. Hence, $1 \neq G^{\mathfrak{x}} \leq P$. If all subgroups of $G^{\mathfrak{3}}$ of order $p$ or order 4 are normal in $G$, then $\Omega\left(G^{\Im}\right) \leq Z_{\mathfrak{U}}(G)$ (see the above Remark). Since $\mathfrak{U}$ and $\mathfrak{I}$ are saturated formations with $\mathfrak{U} \subseteq \mathfrak{\Im}$, it follows that $Z_{\mathfrak{U}}(G) \leq Z_{\mathfrak{\Im}}(G)$ (see [5, IV, 3.11]). Hence $\Omega\left(G^{\Im}\right) \leq Z_{\Im}(G)$. Applying Lemma 2.4, $G \in \Im$; a 
contradiction. Thus, there exists a subgroup $H$ of $G^{\Im}$ of order $p$ or order 4 such that $H$ is not normal in $G$. By hypothesis, $H$ is c-normal in $G$. Then there exists a normal subgroup $N$ of $G$ such that $G=H N$ with $H \cap N \leq H_{G}$, and since $H$ is not normal in $G$, it follows that $N<G$. Clearly, $G^{\Im} \leq N$. Since $G / N$ is a $p$-group, it follows that $G / N \in \mathfrak{U} \subseteq \mathfrak{I}$. Hence, $G^{\Re} \leq N$; a final contradiction.

Below we list some immediate corollaries of Theorem 3.2.

COROLlaRY 3.3 (Wang [11, Theorem 4.2, page 964]). If all subgroups of $G$ of prime order or order 4 are c-normal in $G$, then $G$ is supersolvable.

COROLLARY 3.4. If all subgroups of a group $G$ of prime order are c-normal in $G$, then $G$ is supersolvable if and only if $G$ is $p$-nilpotent, where $p$ is the smallest prime dividing $|G|$.

COROLLARY 3.5. If $G$ is a solvable group and all subgroups of Fit(G) of prime order or order 4 are c-normal in $G$, then $G$ is supersolvable.

PROOF. We prove the result by induction on $|G|$. If all subgroups of Fit $(G)$ of prime order or order 4 are normal in $G$, then $G$ is supersolvable by Lemma 2.5. Thus, we may assume that there exists a subgroup $H$ of Fit $(G)$ of prime order or order 4 such that $H$ is not normal in $G$. By hypothesis, $H$ is c-normal in $G$. Then there exists a normal subgroup $N$ of $G$ such that $G=H N$ with $H \cap N \leq H_{G}$, and since $H$ is not normal in $G$, it follows that $N<G$. Clearly, $G=\operatorname{Fit}(G) N$ and $\operatorname{Fit}(N)<\operatorname{Fit}(G)$. By Lemma 2.1 (i), all subgroups of Fit $(N)$ of prime order or order 4 are c-normal in $N$. Then, by induction on $|G|, N$ is supersolvable. Since $G / \operatorname{Fit}(G) \cong N /(N \cap \operatorname{Fit}(G))$ is supersolvable, it follows by Theorem 3.2 , that $G$ is supersolvable.

The following example shows that the converse of Corollary 3.3, is not true.

EXAMPLE. Let $C_{n}$ be a cyclic group of order $n$. Consider the wreath product $G=C_{9} r w r C_{2}$. Then $|G|=\left|C_{2} \| C_{9}\right|^{2}$ and so $G$ is supersolvable. It is easy to check that $\Phi(G)$ contains a subgroup $H$ of order 3 that fails to be normal in $G$ and hence $H$ is not c-normal in $G$. The same example shows that the converse of Corollary 3.5, is not true.

We are now ready to prove:

THEOREM 3.6. Let $\mathfrak{I}$ be a saturated formation containing $\mathfrak{U}$ and let $G$ be a group. Then the following two statements are equivalent:

(i) $G \in \Im$. 
(ii) There exists a normal solvable subgroup $K$ in $G$ such that $G / K \in \Im$ and all subgroups of $\operatorname{Fit}(K)$ of prime order or order 4 are c-normal in $G$.

ProOF. (i) implies (ii): If $G \in \Im$, then (ii) is true with $K=1$.

(ii) implies (i): Suppose the result is false and let $G$ be a counterexample of minimal order. By Lemma 2.1 (i) and Corollary 3.5, $K$ is supersolvable. Then by [12, Theorem 1.8, page 6], $K$ possesses an ordered Sylow tower and so $K$ has a normal Sylow $p$-subgroup $P$, where $p$ is the largest prime dividing $|K|$. Clearly, $P$ is a normal $p$-subgroup of $G$. If all subgroups of $P$ of order $p$ or order 4 are normal in $G$, then $\Omega(P) \leq Z_{\mathfrak{U}}(G)$. Since $\mathfrak{U}$ and $\mathfrak{\Im}$ are saturated formations with $\mathfrak{U} \subseteq \Im$, it follows that $Z_{\mathfrak{U}}(G) \leq Z_{\mathfrak{I}}(G)$ (see [5, IV, 3.11]). Hence $\Omega(P) \leq Z_{\mathfrak{\Im}}(G)$. By Lemma 2.6, $G / C_{G}(\Omega(P)) \in \Im$ and since $G / K \in \mathfrak{J}$, it follows that $G / C_{K}(\Omega(P)) \in \Im$. Let $V$ be a Sylow $p$-subgroup of $C_{K}(\Omega(P))$. Clearly, $\Omega(V) \leq \Omega(P) \leq Z_{\mathfrak{Y}}(G)$. Then by Lemma 2.4, $G / O_{p^{\prime}}\left(C_{K}(\Omega(P))\right) \in \Im$ and since $O_{p^{\prime}}\left(C_{K}(\Omega(P))\right) \leq O_{p^{\prime}}(K)$, it follows that $G / O_{p^{\prime}}(K) \in \Im$. Then

$$
(G / P) /\left(O_{p^{\prime}}(K) P / P\right) \cong G / O_{p^{\prime}}(K) P \cong\left(G / O_{p^{\prime}}(K)\right) /\left(O_{p^{\prime}}(K) P / O_{p^{\prime}}(K)\right) \in \Im
$$

Put Fit $\left(O_{p^{\prime}}(K) P / P\right)=L / P$. Clearly, $L=P\left(L \cap O_{p^{\prime}}(K)\right)$ and so $L / P \cong L \cap O_{p^{\prime}}(K)$ is nilpotent. Since $P$ and $L \cap O_{p^{\prime}}(K)$ are normal nilpotent subgroups of $K$, it follows that $L=P\left(L \cap O_{p^{\prime}}(K)\right)$ is a normal nilpotent subgroup of $K$. Then $L \leq \operatorname{Fit}(K)$ and so $\operatorname{Fit}\left(O_{p^{\prime}}(K) P / P\right)=\operatorname{Fit}(K) / P$. Hence, by Lemma 2.2, all subgroups of Fit $\left(O_{p^{\prime}}(K) P / P\right)$ of prime order or order 4 are c-normal in $G / P$. By the minimality of $G, G / P \in \Im$. Then by Theorem 3.2, $G \in \mathfrak{\Im}$; a contradiction. Thus, there exists a subgroup $H$ of $P$ of order $p$ or order 4 such that $H$ is not normal in $G$. By hypothesis, $H$ is c-normal in $G$. Then there exists a normal subgroup $N$ of $G$ such that $G=H N$ with $H \cap N \leq H_{G}$ and since $H$ is not normal in $G$, it follows that $N<G$. Clearly, $G=P N=K N$ and so $G / K \cong N /(N \cap K) \in \Im$. Since $N \cap K$ is a normal subgroup of $K$, it follows that $\operatorname{Fit}(N \cap K) \leq \operatorname{Fit}(K)$. Hence, by Lemma 2.1 (i), all subgroups of Fit $(N \cap K)$ of prime order or order 4 are c-normal in $N$. By the minimality of $G$, $N \in \Im$. Since $G / P \cong N /(N \cap P) \in \Im$, it follows by Theorem 3.2, that $G \in \Im$; a final contradiction.

Finally we prove the following result:

THEOREM 3.7. Let $\Im$ be a saturated formation containing $U$ and let $G$ be a solvable group. Then the following two statements are equivalent:

(i) $G \in \mathfrak{\Im}$.

(ii) There exists a normal subgroup $K$ in $G$ such that $G / K \in I$ and all maximal subgroups of all Sylow subgroups of $\mathrm{Fit}(K)$ are c-normal in $G$. 
ProOf. (i) implies (ii): If $G \in \mathfrak{I}$, then (ii) is true with $K=1$.

(ii) implies (i): Suppose the result is false and let $G$ be a counterexample of minimal order. We separate the proof into two cases:

Case $1 . K \cap \Phi(G) \neq 1$. Then there exists a prime $p$ such that $p$ divides $|K \cap \Phi(G)|$. Let $P$ be a Sylow $p$-subgroup of $K \cap \Phi(G)$. Clearly, $P$ is a normal $p$-subgroup of $G$ and so $(G / P) /(K / P) \cong G / K \in \Im$. By [6, Satz 3.5, page 270], $\operatorname{Fit}(K / P)=\operatorname{Fit}(K) / P$. Then by Lemma 2.1 (ii) and Lemma 2.2, all maximal subgroups of all Sylow subgroups of Fit $(K / P)$ are c-normal in $G / P$. By the minimality of $G, G / P \in \Im$. Since $P \leq \Phi(G)$ and $\Im$ is a saturated formation, it follows that $G \in \Im$; a contradiction.

Case 2. $K \cap \Phi(G)=1$. If all maximal subgroups of all Sylow subgroups of $\operatorname{Fit}(K)$ are normal in $G$, then $G \in \mathfrak{I}$ by Lemma 2.7 ; a contradiction. Thus, there exists a maximal subgroup $P_{1}$ of a Sylow $p$-subgroup $P$ of $F i t(K)$, for some prime $p$, such that $P_{1}$ is not normal in $G$. By hypothesis, $P_{1}$ is c-normal in $G$. Then there exists a normal subgroup $H$ of $G$ such that $G=P_{1} H$ with $P_{1} \cap H \leq\left(P_{1}\right)_{G}$, and since $P_{1}$ is not normal in $G$, it follows that $H<G$. Let $M$ be a maximal subgroup of $G$ such that $H \leq M<G$. Then $M$ is a normal subgroup of $G$ as $G / H$ is a $p$-group and so $G=P_{1} M=P M$. Since $P \cap \Phi(G)=K \cap \Phi(G)=1$, it follows by Lemma 2.8, that $P=R_{1} \times R_{2} \times \cdots \times R_{n}$, where $R_{i}$ is a minimal normal subgroup of $G$, for every $1 \leq i \leq n$. Then $R_{i} \not \leq M$, for some $i$. Hence, $G=R_{i} M$ and $R_{i} \cap M=1$. Clearly, $\left(G / R_{i}\right) /\left(K / R_{i}\right) \cong G / K \in \Im$. Put Fit $\left(K / R_{i}\right)=L / R_{i}$. Since $R_{i} \leq L \leq R_{i} M=G$, it follows that $L=R_{i}(L \cap M)$ and so $L / R_{i} \cong L \cap M$ is nilpotent. Since $R_{i}$ and $L \cap M$ are normal nilpotent subgroups of $G$, it follows that $L=R_{i}(L \cap M)$ is a normal nilpotent subgroup of $G$. Then $L=\operatorname{Fit}(K)$ and so $\operatorname{Fit}\left(K / R_{i}\right)=\operatorname{Fit}(K) / R_{i}$. Hence, by Lemma 2.1 (ii) and Lemma 2.2, all maximal subgroups of all Sylow subgroups of Fit $\left(K / R_{i}\right)$ are c-normal in $G / R_{i}$. By the minimality of $G, G / R_{i} \in \Im$. Since $G / M \cong R_{i} \in \mathfrak{U} \subseteq \Im$, it follows that $G \cong G /\left(R_{i} \cap M\right) \in \mathfrak{\Im}$; a final contradiction.

REMARKS. (i) Our results are not true for saturated formations which do not contain $\mathfrak{U}$. For example, if $\Im$ is the saturated formation of all nilpotent groups, then the symmetric group of degree three is a counterexample.

(ii) Our results are not true for non-saturated formations. Let $\mathfrak{\Im}$ be the formation composed of all groups $G$ such that $G^{\mathfrak{U}}$, the supersolvable residual, is elementary abelian. Clearly, $\mathfrak{U} \subseteq \Im$ but $\Im$ is not saturated. Put $G=S L(2,3)$ and $K=Z(G)$. Then $G / K$ is isomorphic to the alternating group of degree four and so $G / K \in \mathfrak{I}$, but $G$ does not belong to $\Im$.

(iii) Theorem 3.2 is not true in general if we replace the condition 'prime order or order 4' by 'prime order', as the following example shows. The class $\mathfrak{I}=\mathfrak{A} \mathfrak{U}$ of groups whose derived subgroup is nilpotent is a saturated formation containing the class $\mathfrak{U}$ of supersolvable groups (see [6, VI, 9.1 (b)]). Consider the group $G=$ $G L(2,3)$. This group has a normal subgroup $K$ isomorphic to to the quaternion 
group of order 8 such that $G / K$ is isomorphic to the symmetric group of degree 3 . Therefore we have that $G / K \cong \Im$. Notice that the unique subgroup of $K$ with prime order is $Z(K)$ and this is not only a $c$-normal subgroup of $G$. But the derived group $G^{\prime}=S L(2,3)$ is not nilpotent, and then $G \notin \Im$. Since $K$ is a nilpotent group, the same example shows Theorem 3.6 is not true in general if we require that all subgroups of Fit $(K)$ of prime order are $c$-normal in $G$.

(iv) Theorems 3.6 and 3.7 are not true if we omit the condition of solvability. Put $G=H \times K$, where $H \in \mathfrak{U}$ and $K=S L(2,5)$. Then $\mid$ Fit $(K) \mid=2$ and $G / K \cong H \in \mathfrak{U}$, but $G$ does not belong to $\mathfrak{U}$.

\section{References}

[1] M. Asaad, 'On maximal subgroups of Sylow subgroups of finite groups', Comm. Algebra 26 (1998), 3647-3652.

[2] M. Asaad and P. Csörgö, 'The influence of minimal subgroups on the structure of finite groups', Arch. Math. 72 (1999), 401-404.

[3] J. B. Derr, W. E. Deskins and N. P. Mukherjee, 'The influence of minimal p-subgroups on the structure of finite groups', Arch. Math. 45 (1985), 1-4.

[4] L. Deyu and G. Xiuyun, 'The influence of c-normality of subgroups on the structure of finite groups II', Comm. Algebra 26 (1998), 1913-1922.

[5] K. Doerk and T. Hawkes, Finite soluble groups (Walter De Gruyter, Berlin, 1992).

[6] B. Huppert, Endliche Gruppen I (Springer, Berlin, 1967).

[7] —_ 'Zur Theorie der Formationen', Arch. Math. 19 (1968), 561-574.

[8] N. Ito, 'Uber eine zur Frattini. Gruppe duale Bildung', Nagoya Math. J. 9 (1955), 123-127.

[9] O. H. Kegel, 'Sylow-Gruppen und Subnormalteiler endlicher Gruppen', Math. Z. 78 (1962), 205221.

[10] A. Shaalan, 'The influence of S-quasinormality of some subgroups on the structure of a finite group', Acta Math. Hungar. 56 (1990), 287-293.

[11] Y. Wang, 'C-normality of groups and its properties', J. Algebra 180 (1996), 954-965.

[12] M. Weinstein (ed.), Between nilpotent and solvable (Polygonal Publ. H., Washington, 1982).

[13] G. Xiuyun and L. Deyu, 'The influence of c-normality of subgroups on the structure of finite groups', J. Pure Appl. Algebra 150 (2000), 53-60.

\section{Cairo University}

Faculty of Science

Department of Mathematics

Giza

Egypt

e-mail: moasmohs@frcu.eun.eg 